www.nature.com/jhg

\title{
Warfarin pharmacogenetics: development of a dosing algorithm for Omani patients
}

\author{
Anil Pathare ${ }^{1}$, Murtadha Al Khabori ${ }^{1}$, Salam Alkindi ${ }^{1,2}$, Shoaib Al Zadjali ${ }^{1}$, Rhea Misquith ${ }^{1}$, Hammad Khan ${ }^{1}$, \\ Claudine Lapoumeroulie ${ }^{3}$, Andras Paldi ${ }^{4}$ and Rajagopal Krishnamoorthy ${ }^{3}$
}

The objective of our present study was to develop a warfarin dosing algorithm for the Omani patients, as performances of warfarin dosing algorithms vary across populations with impact on the daily maintenance dose. We studied the functional polymorphisms of CYP2C9, CYP4F2 and VKORC1 genes to evaluate their impact on the warfarin maintenance dose in an admixed Omani patient cohort with Caucasian, African and Asian ancestries. We observed a 64-fold inter-patient variability for warfarin to achieve stable international normalized ratio in these patients. Univariate analysis revealed that age, gender, weight, atrial fibrillation, deep vein thrombosis/pulmonary embolism and variant genotypes of CYP2C9 and VKORC1 loci were significantly associated with warfarin dose in the studied patient population. However, multiple regression model showed that only the atrial fibrillation, and homozygous CYP2C9 variant genotypes $\left({ }^{*} 2 /{ }^{*} 3\right.$ and $\left.{ }^{*} 3 /{ }^{*} 3\right)$ and VKORC1 GA and AA genotypes remained significant. A multivariate model, which included demographic, clinical and pharmacogenetic variables together explained $63 \%$ of the overall inter-patient variability in warfarin dose requirement in this microgeographically defined, ethnically admixed Omani patient cohort on warfarin. This locally developed model performed much better than the International Warfarin Pharmacogenetics Consortium (IWPC) model as the latter could only explain $34 \%$ of the inter-patient variability in Omani patients. VKORC1 3673G $>$ A polymorphism emerged as the single most important predictor of warfarin dose variability, even in this admixed population (partial $R^{2}=0.45$ ).

Journal of Human Genetics (2012) 57, 665-669; doi:10.1038/jhg.2012.94; published online 2 August 2012

Keywords: admixture; CYP2C9; CYP4F2; resistance; sensitive; VKORC1; Warfarin

\section{INTRODUCTION}

Several dosing algorithms have been developed to predict the appropriate patient-specific warfarin dose for various population groups in recent years. ${ }^{1-4}$ Most of these algorithms, in a multivariate regression analysis incorporate age, gender, body weight/surface area, co-medications, co-morbidities and functionally relevant genetic polymorphisms of CYP2C9, CYP4F2 and VKORC1 loci as covariates to estimate their contribution to individual warfarin dose.

The performance of these dosing algorithms varies across populations or cohorts, and the concept of their 'population/cohort specificity' has emerged. ${ }^{5}$ The inconsistent performance of these dosing algorithms across population groups may reflect significant differences in the contribution of non-genetic factors, including compliance rate or yet to be uncovered additional genetic variants or factors. It implies that performance must be assessed for each study population before making therapeutic decisions, especially for admixed populations. ${ }^{4,5}$

Towards this end, we carried out a warfarin pharmacogenetic study, in a microgeographically defined, ethnically admixed Omani patients on warfarin therapy, but sharing among them comparable lifestyle and environmental factors.

\section{MATERIALS AND METHODS}

Patients

The study cohort was prospectively recruited at the Sultan Qaboos University Hospital, Muscat, Oman, after approval by the institutional review board and after obtaining written informed consent. Subjects included are consecutive adult patients who were started on warfarin for arterial or venous thrombosis $(n=56)$, non-ischemic heart disease like atrial fibrillation $(n=115)$, cardiac prosthesis $(n=36)$ or other indications like antiphospholipid syndrome, paraparesis or dilated cardiomyopathy $(n=5)$. Exclusion criteria included the presence of severe co-morbidities like liver cirrhosis, uremia or malignant diseases.

A total of 212 patients (113 males and 99 females) of Omani ethnicity (since at least three to four generations) were analyzed in the present study to derive the dosing algorithm after exclusion of two patients with confirmed VKORC1 exon mutation (Val66Met mutation) that are known to be associated with dose escalation. Their mean age was 49.8 (s.d. \pm 18.1 ) years. Their demographic data, indication for anticoagulant therapy, target international normalized ratio (INR), concomitant disease, concomitant medications and dietary information were recorded. All patients were individually monitored and the warfarin maintenance dose for stable anticoagulation was adjusted to achieve a target INR between 2 and 3 based on the American College of Chest Physicians guidelines for warfarin therapy. ${ }^{6} \mathrm{~A}$ patient was considered to have a stable INR when his/her INR was between 2 and 3 on at least 3 consecutive assessments, 3 months after initiating the therapy.

${ }^{1}$ Department of Haematology, Sultan Qaboos University Hospital, Muscat, Oman; ${ }^{2}$ College of Medicine and Health Sciences, SQU, Muscat, Oman; ${ }^{3}$ INSERM, UMR_S 763, Hopital Robert Debre, Paris, France and ${ }^{4}$ Inserm, U951, Ecole Pratique des Hautes Etudes, UMRS_951, Genethon, Evry, France 


\section{DNA studies}

A 5-ml blood sample was collected in tubes containing EDTA. Genomic DNA was isolated using the semi-automated ABI PRISM 6100 Nucleic Acid Prep Station, (Applied Biosystems, Foster City, CA, USA) and samples were stored at $-20{ }^{\circ} \mathrm{C}$ pending analysis. All DNA sequence polymorphisms were studied by direct sequencing of the relevant genome segments amplified by PCR on an ABI PRISM 3100 Genetic Analyzer (Applied Biosystems) using customdesigned primers (available on request).

\section{Genotyping}

CYP2C9 and CYP4F2. The DNA segments encompassing the exons 3 and 7 of CYP2C9 genes and the exon 11 of CYP4F2 genes were amplified by PCR, and the PCR products were submitted for DNA sequencing to explore the following single-nucleotide polymorphisms (SNPs); CYP2C9 ${ }^{\star 2}$ (rs1799853), *3 (rs1057910), and CYP4F2V433M or ${ }^{*} 3$ (rs2108622), respectively, using a sequencing protocol provided by the manufacturer (Applied Biosystems).

VKORC1. The DNA segments containing the following SNPs of the VKORC1 locus 3673G $>$ A (rs9923231), 5808T $>$ G (rs 2884737), 6009C $>\mathrm{T}$ (rs 17708472), $6484 \mathrm{C}>\mathrm{T}$ (rs 9934438) and 9041G > A (rs 7294) (GeneBank accession number AY587020) $)^{7,8}$ were PCR-amplified and analyzed by DNA sequencing of the PCR product.

\section{Statistical analysis}

We randomly chose $70 \%$ of the enrolled patients in a 'derivation cohort ( $n=142$ ) for developing the dose-prediction model. The remaining $30 \%$ of our patients $(n=70)$ constituted the 'validation cohort' and was used for testing the finally selected regression model from the derivation cohort. Least-square linear regression analysis was performed to derive the pharmacogenetic model using STATA ver. 11.1 (StataCorp, College Station, TX, USA). The distribution of warfarin daily maintenance was skewed and was log-transformed to achieve constant variance and normality. We performed univariate linear regression on the following variables: simvastatin, amiodarone, hypertension, diabetes, atrial fibrillation, deep vein thrombosis, mechanical valve, age, weight, gender,

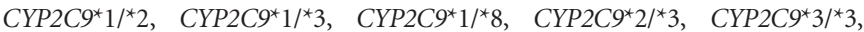

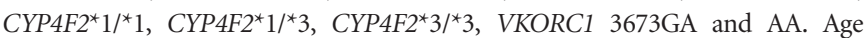
and weight were entered as linear variables for gender $0=$ female and $1=$ male, whereas all other covariates in the model were entered as dummy variables $0=$ absent and $1=$ present. Missing parameters/values (for example, PCR failures in genotype determination) were treated as 'missing' in the model. Multivariate linear regression was used to derive our final model and included all significant covariates from the univariate linear regression with a $P$-value $\leqslant 0.05$. Outlier observations were defined as any observation with studentized residual of greater than \pm 2 s.d. and were removed from the univariate and multivariate linear regression analyses. The partial $R^{2}$ statistic was used to characterize the contribution of each variable to the model. It measures the degree of association between two random variables. This was used to evaluate the accuracy of the model's predictability. Furthermore, the algorithm developed with multivariate linear regression in the derivation cohort (142 patients) was assessed with Pearson's correlation analysis in the validation cohort (70 patients) and the mean absolute error (MAE), that is, the mean of the predicted and actual maintenance doses using the validation cohort.

\section{RESULTS}

Linkage disequilibrium

The VKORC1 promoter polymorphism 3673G > A (rs9923231) was in complete linkage disequilibrium with the intron 1 polymorphism $6484 \mathrm{C}>\mathrm{T}$ (rs 9934438) in this Omani cohort.

\section{Impact of CYP2C9 and VKORC1 polymorphisms on stable warfarin dose}

The median daily warfarin dose in $C Y P 2 C 9{ }^{\star} 1 /{ }^{\star} 1$ patients was 5.0 with the interquartile range between 3.0 and $6.0 \mathrm{mg}$, whereas in the VKORC1 3673GG patients, it was 5.5 with interquartile range between 4.5 and $7.0 \mathrm{mg}$. The daily warfarin dose $(\mathrm{mg})$ ranged from 0.5 to 32 , (a 64-fold inter-individual variability). The daily warfarin dose was significantly lower in $C Y P 2 C 9^{\star} 2 /{ }^{*} 3$ or $C Y P 2 C 9{ }^{*} 3{ }^{*} 3$, or $V K O R C 1$ $3673 \mathrm{AA}$, with the median dose being $3.0,1.0$ and $2.0 \mathrm{mg}$ per day, respectively $(P<0.05$; Student-Newman-Keuls test) (Figure 1$)$.

\section{Warfarin dosing algorithm}

The demographic, clinical and genetic variables in the derivation and validation cohorts are shown in Table 1 . Genotype quality control was performed on $10 \%$ of all the patient samples with an overall concordance of $100 \%$ in the CYP2C9 and VKORC1 SNPs, which also satisfied the Hardy-Weinberg distribution.

The initial univariate analysis showed that the age, gender, weight, atrial fibrillation, deep vein thrombosis/pulmonary embolism, CYP2C9 and VKORC1 variant allele/genotype were significantly associated with the daily warfarin dose $(P<0.05)$ (Table 2$)$. All the above significant variables were then entered into a multiple regression model, which showed that only the atrial fibrillation, homozygous CYP2C9 variant genotypes $\left({ }^{*} /{ }^{*} 3\right.$ or $\left.{ }^{*} 3 / * 3\right)$ and $V K O R C 1$ 3673GA and AA genotypes remained significant (Table 3 ). On the basis of the coefficient of regression estimates, the proposed regression equation for predicting the stable daily warfarin dose for the Omani patients was: warfarin daily dose $(\mathrm{mg})=\exp [0.69-(0.001 \times$ age in years $)+(0.0008 \times$ weight in $\mathrm{Kg})+0.034 \times(1$, if gender male, else 0$)+0.0123 \times(1$, deep vein thrombosis, else 0$)+0.102 \times(1$, if Atrial Fibrillation, else, 0$)-0.036 \times\left(1\right.$, if $C Y P 2 C 9^{\star} 1 /{ }^{\star} 2$, else 0$)-0.088 \times$ $\left(1\right.$, if $C Y P 2 C 9^{\star} 11^{*} 3$, else 0$)-0.117 \times\left(1\right.$, if $C Y P 2 C 9^{\star} 1 /{ }^{*} 8$, else 0$)$ $-0.240 \times\left(1\right.$, if $C Y P 2 C 9^{*} 2 /^{*} 3$, else 0$)-0.482 \times\left(1\right.$, if $C Y P 2 C 9^{\star} 3 /^{*} 3$, else 0$)-0.164 \times(1$, if VKORC1 3673GA, else 0$)-0.404 \times(1$, if VKORC1 3673 AA, else 0)]. This full model accounted for 63\% of the total variance in warfarin dose requirement in our derivation cohort.

\section{Validation of the regression model derived from the derivation cohort}

We analyzed the validation cohort to test the regression equation obtained from the derivation cohort. Figure 2 shows the comparison of the observed log of stable warfarin dose in the validation cohort to the warfarin dose predicted by the regression equation obtained from the derivation cohort. The Pearson's correlation was 0.5825

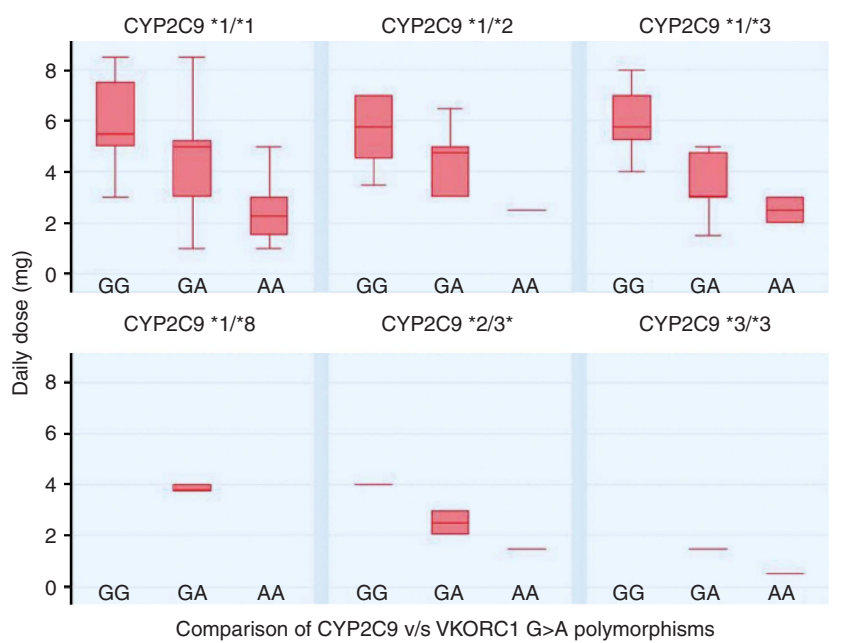

Figure 1 Box-Whisker plot showing the correlation of CYP2C9 and VKORC1 $\mathrm{G}>\mathrm{A}$ polymorphisms (abscissa) with daily warfarin dose (ordinate). CYP2C9 genotype is labeled as wild-type $\left({ }^{*} 1 /{ }^{*} 1\right)$, one variant allele $\left({ }^{*} 1 /{ }^{*} 2,{ }^{*} 1 /{ }^{*} 3\right.$, ${ }^{*} 1 / * 8$ ), or two variant alleles $\left(* 2 / * 3,{ }^{*} 3 / * 3\right)$; and within each CYP2C9 genotype, the VKORC1 3673 genotype is shown as GG, GA or AA. 
Table 1 Demographic and clinical characteristics of the derivation and validation cohorts

\begin{tabular}{|c|c|c|c|}
\hline Variables & $\begin{array}{c}\text { Derivation }(\mathrm{n}=142) \\
\text { cohort }\end{array}$ & $\begin{array}{c}\text { Validation }(\mathrm{n}=70) \\
\text { cohort }\end{array}$ & P-value \\
\hline \multicolumn{4}{|l|}{ Warfarin dose (mg per day) } \\
\hline Median & 4.75 & 4.75 & n.s. \\
\hline Interquartile range & $3-5.5$ & $3-5.9$ & \\
\hline Age, mean (s.d.), year & $51.5(17.7)$ & $48.2(18.6)$ & n.s. \\
\hline \multicolumn{4}{|l|}{ Gender } \\
\hline Male, $N(\%)$ & $81(57)$ & $32(46)$ & n.s. \\
\hline Female, $N(\%)$ & $61(43)$ & $38(54)$ & n.s. \\
\hline Weight, mean (s.d.), kg & $68.5(18.2)$ & $69.2(18.7)$ & n.s. \\
\hline Hypertension, N (\%) & $35(25)$ & $25(36)$ & n.s. \\
\hline $\mathrm{DM}, N(\%)$ & $28(20)$ & $17(24)$ & n.s. \\
\hline Simvastatin, $N(\%)$ & $32(23)$ & $15(22)$ & n.s. \\
\hline Amiodarone, $N(\%)$ & $8(7)$ & $3(4)$ & n.s. \\
\hline $\mathrm{AF}, N(\%)$ & $77(54)$ & $38(54)$ & n.s. \\
\hline DVT/PE, $N(\%)$ & $31(22)$ & $25(36)$ & n.s. \\
\hline Mechanical valve, $N(\%)$ & $25(18)$ & $11(16)$ & n.s. \\
\hline CYP2C9, N(\%) & $139(98)$ & 69 (99) & n.s. \\
\hline${ }^{*} 1 /{ }^{*} 1, N(\%)$ & $106(75)$ & $50(71)$ & n.s. \\
\hline${ }^{*} 1 / * 2, N(\%)$ & $17(12)$ & $7(10)$ & n.s. \\
\hline${ }^{*} 1 / * 3, N(\%)$ & $9(6)$ & $9(13)$ & n.s. \\
\hline${ }^{*} 1 / * 8, N(\%)$ & $1(1)$ & $2(3)$ & n.s. \\
\hline$* 2 / * 3, N(\%)$ & $4(3)$ & $1(1)$ & n.s. \\
\hline$* 3 / * 3, N(\%)$ & $2(2)-$ & - & \\
\hline Missing, $N(\%)$ & $3(2)$ & $1(1)$ & n.s. \\
\hline CYP4F2, N (\%) & $129(91)$ & $62(89)$ & n.s. \\
\hline Missing, $N(\%)$ & $13(9)$ & $8(11)$ & n.s. \\
\hline VKORC1_3673, N (\%) & $133(94)$ & $66(94)$ & n.s. \\
\hline $\mathrm{GG}, N(\%)$ & $55(39)$ & $30(43)$ & n.s. \\
\hline $\mathrm{GA}, N(\%)$ & $56(39)$ & $29(41)$ & n.s. \\
\hline $\mathrm{AA}, N(\%)$ & $22(15)$ & $7(10)$ & n.s. \\
\hline Missing, $N(\%)$ & $9(6)$ & $4(6)$ & n.s. \\
\hline VKORC1_5808, N (\%) & $116(82)$ & $59(84)$ & n.s. \\
\hline Missing, $N(\%)$ & $26(18)$ & $11(16)$ & n.s. \\
\hline VKORC1_6009, N (\%) & $121(85)$ & $62(89)$ & n.s. \\
\hline Missing, $N(\%)$ & $21(15)$ & $8(12)$ & n.s. \\
\hline VKORC1_9041, N (\%) & $119(84)$ & $62(89)$ & n.s. \\
\hline Missing, $N(\%)$ & $23(16)$ & $8(12)$ & n.s. \\
\hline
\end{tabular}

Abbreviations: AF, atrial fibrillation; DM, diabetes mellitus; DVT/PE, deep vein thrombosis/ pulmonary embolism; n.s., not significant.

n.s., $P>0.05$

$(P<0.0001)$ and suggested a good correlation between the derivation and the validation cohort (both matched for the demographic, clinical and genetic characteristics; $P>0.05)$. Furthermore, the validation cohort could predict warfarin dose with the average MAE of $-0.26 \pm 01.9 \mathrm{mg}$ per day of the derivation cohort.

Testing the International Warfarin Pharmacogenetics Consortium (IWPC) algorithm on our Omani cohort

We used our patient cohort data to assess the predictive power of the previously published algorithm by the IWPC $^{2}$ (Figure 3). The adjusted $R^{2}$ was only 0.336 (constant \pm s.e.; $1.56 \pm 0.331 ; 95 \%$
Table 2 Univariate analysis of covariates associated with the interindividual variability of warfarin dose (derivation cohort, $n=142$ )

\begin{tabular}{|c|c|c|c|c|c|c|}
\hline Univariate $^{a}$ & $\mathrm{n}$ & Constant & Coefficient & +s.e. & P-values & Adjusted $\mathrm{R}^{2}$ \\
\hline Age & 142 & 0.73 & -0.002 & +0.0009 & 0.039 & 0.025 \\
\hline Gender & 142 & 0.57 & 0.142 & +0.0326 & 0.00 & 0.122 \\
\hline Weight & 142 & 0.40 & 0.003 & +0.0009 & 0.00 & 0.090 \\
\hline Hypertension & 35 & 0.66 & -0.021 & +0.0396 & 0.59 & -0.006 \\
\hline DM & 28 & 0.52 & 0.059 & +0.0442 & 0.19 & 0.006 \\
\hline$A F$ & 77 & 0.44 & 0.127 & +0.0319 & 0.00 & 0.104 \\
\hline DVT/PE & 31 & 0.82 & -0.114 & +0.0358 & 0.002 & 0.065 \\
\hline Mechanical valve & 25 & 0.64 & -0.009 & +0.0449 & 0.83 & -0.007 \\
\hline Simvastatin & 32 & 0.54 & 0.048 & +0.0426 & 0.26 & 0.002 \\
\hline Amiodarone & 08 & 0.49 & 0.071 & +0.0725 & 0.33 & -0.0004 \\
\hline CYP2C9 & 139 & 0.93 & -0.026 & +0.005 & 0.00 & 0.165 \\
\hline CYP4F2 & 129 & 0.62 & -0.0002 & +0.003 & 0.95 & -0.008 \\
\hline VKORC1_3673 & 133 & 0.97 & -0.202 & +0.019 & 0.00 & 0.452 \\
\hline VKORC1_5808 & 116 & 0.85 & -0.119 & +0.024 & 0.00 & 0.175 \\
\hline VKORC1_6009 & 121 & 0.54 & 0.076 & +0.029 & 0.01 & 0.050 \\
\hline VKORC1_9041 & 119 & 0.47 & 0.110 & +0.030 & 0.00 & 0.103 \\
\hline
\end{tabular}

Abbreviations: AF, atrial fibrillation; DM, diabetes mellitus; DVT/PE, deep vein thrombosis/ pulmonary embolism.

${ }^{a}$ Age and weight were entered as linear variables; gender $0=$ females, $1=$ males, whereas all other covariates in the model were entered as dummy variables $(0=$ absent, $1=$ present).

confidence interval (CI; 0.903-2.21). Furthermore, predicted dose using the IWPC equation was $13 \%$ higher than the actual dose in our Omani patients (co-efficient $=1.1313$ ).

\section{DISCUSSION}

The present study explores the pharmacogenetic aspects of warfarin in Omani patients, a genetically admixed population with Caucasian, African and Asian ancestries. The objective of the study was to evaluate the influence of genetic variants of CYP2C9, CYP4F2 and VKORC1 loci known to be associated with variable degree of sensitivity/resistance to warfarin. In this study, we analyzed data on 212 patients who were on stable anticoagulation with warfarin (target INR $=2.5$ ), by using a multiple regression analysis approach with genetic (CYP2C9, CYP4F2 and VKORC1), demographic (age, weight and gender) and clinical parameters (diabetes mellitus, hypertension, use of simvastatin and amiodarone) to develop a dosing algorithm that explained $63 \%$ of the total variance of the stable warfarin dose (Table 3). This falls towards the upper end of the reported range of $31-57 \%$ for various population groups. ${ }^{1-4,9-13}$

Contribution of demographic (age, gender and weight) and genetic factors for warfarin dose variance in the Omani patients were 21 and $42 \%$, respectively, of which the VKORC1 $3673 \mathrm{G}>\mathrm{A}$ polymorphism was the most important predictor in our model with a partial $R^{2}$ value of 0.45 in the univariate model. This variant SNP (VKORC1 3673A) located in the promoter region has been shown to affect a transcription factor binding site and result in reduced mRNA expression in human liver tissue. ${ }^{14}$ The predictive power of our algorithm did not improve when the VKORC1 3673G $>$ A was replaced by the other VKORC1 SNPs $(5808 \mathrm{~T}>\mathrm{G}, 6009 \mathrm{C}>\mathrm{T}$ or $9041 \mathrm{G}>\mathrm{A})$ given the strong linkage disequilibrium among them in our population. Limdi et al., ${ }^{1}$ in a recent study demonstrated that VKORC1 $3673 \mathrm{G}>\mathrm{A}$ polymorphism was predictive of warfarin dose across three racial groups, namely Asians, Blacks and Whites, but its contribution varies between 11 and 32\% amongst Caucasians, 30\% in Asians and $4-10 \%$ in African Americans, reflecting its relative frequency in each population. , $^{9,10,15,16}$ 
Table 3 Multivariate analysis of all the covariates that were significant in univariate analysis (derivation cohort, $n=142$ )

\begin{tabular}{|c|c|c|c|c|c|c|}
\hline Covariate & $\mathrm{n}$ & Partial regression & Coefficient s.e. & $95 \% \mathrm{Cl}$ & P-values & $\mathrm{R}^{2}$ \\
\hline Constant & & 0.69 & 0.0898 & (0.52 to 0.87 ) & & \\
\hline Age & 142 & -0.001 & 0.0008 & $(-0.003-0.0004)$ & 0.15 & \\
\hline Gender & 142 & 0.034 & 0.0279 & $(-0.021-0.089)$ & 0.22 & \\
\hline Weight & 142 & 0.0008 & 0.0007 & $(-0.0006$ to 0.002$)$ & 0.24 & \\
\hline $\mathrm{AF}$ & 77 & 0.1015 & 0.035 & (0.0321 to 0.170$)$ & 0.005 & \\
\hline DVT/PE & 31 & 0.0123 & 0.0379 & $(-0.062$ to 0.087$)$ & 0.74 & \\
\hline CYP $2 C 9 * 1 / * 2$ & 17 & -0.0362 & 0.0403 & $(-0.116$ to 0.043$)$ & 0.37 & \\
\hline CYP $2 C 9 * 1 / * 3$ & 09 & -0.0881 & 0.0527 & $(-0.193$ to 0.0165$)$ & 0.09 & \\
\hline CYP 2 C9 $^{*} 1 / * 8$ & 01 & -0.1165 & 0.1501 & $(-0.414$ to 0.181$)$ & 0.44 & \\
\hline CYP2C9*2/*3 & 04 & -0.2397 & 0.0861 & $(-0.410$ to -0.069$)$ & 0.006 & \\
\hline CYP $2 C 9 * 3 / * 3$ & 02 & -0.4817 & 0.1051 & $(-0.690$ to -0.273$)$ & 0.000 & \\
\hline VKORC1_3673GA & 56 & -0.1637 & 0.0294 & $(-0.222$ to -0.105$)$ & 0.000 & \\
\hline VKORC1_3673AA & 22 & -0.4041 & 0.0394 & $(-0.482$ to -0.326$)$ & 0.000 & \\
\hline Full model (all variables) & & & & $R^{2}$ & $=$ & 0.6613 \\
\hline & & & & Adjusted $R^{2}$ & $=$ & 0.6246 \\
\hline
\end{tabular}

Abbreviations: AF, atrial fibrillation; DVT/PE, deep vein thrombosis/pulmonary embolism. Gender $0=$ females, $1=$ males.

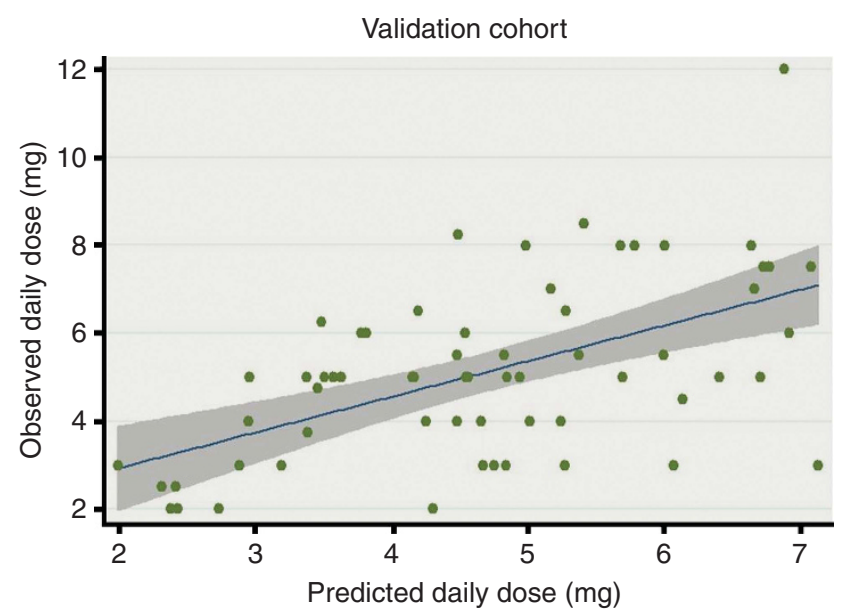

Figure 2 Observed versus predicted stable therapeutic log daily warfarin dose in the validation patient cohort $(n=70)$ in this study. The solid line indicates perfect prediction. The covariates used to build the warfarin dosing algorithm were age, weight, gender, treatment indications atrial fibrillation, deep vein thrombosis/pulmonary embolism, CYP2C9 and VKORC1 3673 genotype.

The contribution of CYP2C9 variant genotypes to the warfarin dosing algorithm was the next significant contributor with a partial $R^{2}$ value of 0.17 in our analysis. CYP2C $9^{\star} 2$ and ${ }^{*} 3$ alleles are the significant variant alleles reported in Caucasians, whereas CYP2C ${ }^{\star} 4$, ${ }^{\star} 5,{ }^{\star} 6,{ }^{\star} 8$ and ${ }^{\star} 11$ are the predominant alleles reported in African Americans. ${ }^{9,15}$ Contribution of the CYP2C 9 variant alleles to warfarin dose variability differed widely across geographical groups collectively classified as Whites (2.5-18.3 mg per day). ${ }^{10,11,15,16}$ Lee et al. ${ }^{17}$ in a study from Singapore, showed that the CYP2C9 genotype accounted for 29, 7 and $0 \%$ of warfarin dose variance among Indians, Chinese and Malay patients, respectively. Cavallari et al..$^{9}$ reported that the influence of CYP2C9 variant alleles on warfarin dose was around 15\% in a predominantly African American population and inclusion of the CYP2C $9{ }^{\star} 5,{ }^{\star} 6,{ }^{\star} 8$ and ${ }^{\star} 11$ alleles further improved the predictive

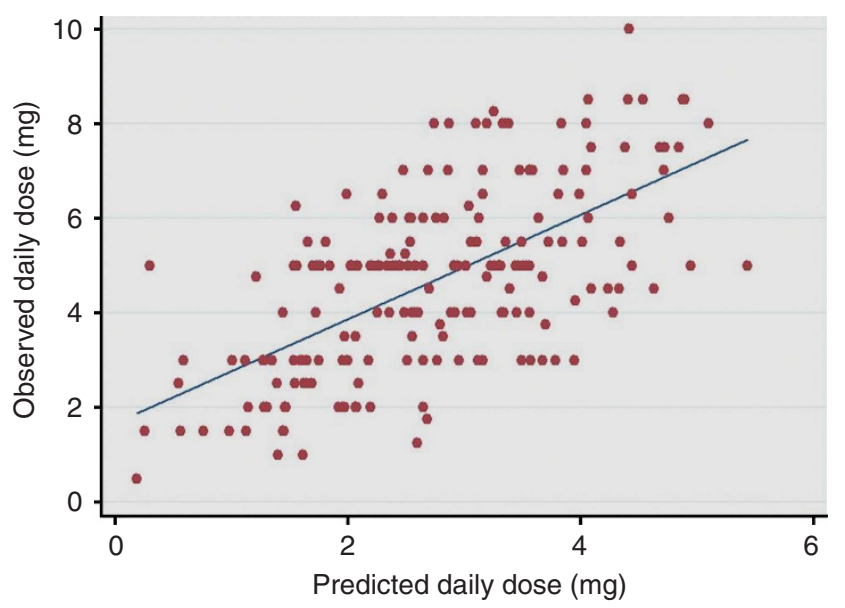

Figure 3 Relationship between daily warfarin dose in Omani patients predicted by inputting the actual warfarin dose used by the Omani study patients in multiple regression model described in the IWPC study ${ }^{2}$ (abscissa, adjusted $R^{2}=0.336$ ) and the observed stable warfarin dose actually taken by the cohort of Omani patients (ordinate, adjusted $\left.R^{2}=0.625\right)$.

ability beyond that seen with $C Y P 2 C 9^{\star} 2$ and ${ }^{\star} 3$. However, despite the gene flow from east Africa into Oman (as demonstrated by the presence of the $C Y P 2 C 9^{\star} 8$ allele), the contribution of $C Y P 2 C 9$ variant alleles remains low in our study cohort.

Our study showed that CYP4F2 variant alleles did not have a significant part in the warfarin dosing algorithm with a partial $R^{2}$ value of -0.008 in our patients. This is in spite of the fact that frequency rate of the minor allele $\left(C Y P 4 F 2^{*} 3\right.$ or $\left.\mathrm{V} 433 \mathrm{M}\right)$ is around $30 \%$ in our population similar to that reported for Caucasian and Asian populations (25-30\%). ${ }^{18}$ The CYP4F2 locus has been shown to code for the primary human liver microsomal vitamin K1 oxidase and the CYP4F2 V433M allele is associated with decreased steadystate hepatic concentrations of the enzyme, with consequent reduction in the metabolism of vitamin $\mathrm{K} 1$ and a higher warfarin requirement for therapeutic anticoagulant effect. ${ }^{19}$ 
The IWPC has collected data in various population groups including Asians, Blacks and Whites across four continents with a pooled population of 6256 chronic warfarin users, and developed a dosing algorithm utilizing demographic, clinical and genetic variables viz CYP2C9 and VKORC1 genotypes. ${ }^{3,4}$ Use of this pharmacogenetic algorithm for estimation of the appropriate initial dose of warfarin is reported to result in dose recommendations that are close to the actual steady-state warfarin dose on continuous therapy for each of the major ethnic groups and for each of the 21 participating sites. ${ }^{2}$ We therefore tested the IWPC dosing algorithm by imputing the actual warfarin dose used by our study cohort in their pharmacogenetic equation (Figure 3) and found that the IWPC algorithm did not perform reasonably well for our admixed Omani population, as it explained only $34 \%$ of the variance in Omani patients (adjusted $R^{2}=0.336$ ) as opposed to $63 \%$ obtained with the locally designed algorithm (the adjusted $R^{2}=0.625$ ). Thus, although the IWPC algorithm has been validated in several individual populations with self-declared racial characteristics, ${ }^{1-3}$ it needs to be emphasized that in Omani patients it did not perform well. Furthermore, the performance of warfarin dosing algorithms in the literature as expressed by correlation coefficient $R^{2}$ vary considerably between 0.2 and 0.6, but is consistently superior in Europeans compared with Asians or populations of African decent. ${ }^{5}$ This discrepancy may arise owing to the fluctuations in racial definitions, clinal variations, social/lifestyle contexts, population migration and historical perspectives. ${ }^{20,21}$ These observations strengthens the notion that warfarin dosing algorithms will be microgeographically defined population-specific.

The potential role of genetic variations at other loci, such as calumenin, gamma glutamyl carboxylase, microsomal epoxide hydrolase and apolipoprotein $\mathrm{E}$, has been proposed by several investigators in the past, but their impact on warfarin dosing algorithm was not demonstrated. ${ }^{9,16,22}$ Furthermore, three genome-wide association studies failed to find any additional genetic loci other than those herein studied (CYP2C9, CYP4F2 and VKORC1) with regard to influence on warfarin dose requirement. ${ }^{23,24}$

In conclusion, in this first report on the impact of genetic, clinical and demographic factors involved in warfarin dose requirement in the Omani patients, a significant percentage of variability still (around $37 \%$ ) awaits explanation. Similar to the Brazilian patients with mixed ethnicities, a general warfarin dosing algorithm like the IWPC performed poorly in this microgeographically defined, ethnically admixed Omani patient. Nevertheless, the VKORC1 3673G $>$ A polymorphism emerged as the single most important determinant of warfarin dose variability in this admixed population (partial $\left.R^{2}=0.45\right)$.

\section{CONFLICT OF INTEREST}

The authors declare no conflict of interest.

\section{ACKNOWLEDGEMENTS}

This study was the result of a collaborative project between INSERM, UMR_S 763, Paris, France, and Department of Hematology, Sultan Qaboos University, Muscat, Oman. It was supported by a research grant (IG/MED/HEME/09/01) from the Sultan Qaboos University. We sincerely thank the College Research Committee of the Sultan Qaboos University for facilitating the study and the University Hospital administration for allowing the use of clinical material.
1 Limdi, N. A., Wadelius, M., Cavallari, L., Eriksson, N., Crawford, D. C., Lee, M. T. et al.; on behalf of the IWPC. Warfarin pharmacogenetics: VKORC1 genotype as predictive as haplotype across three racial groups. Blood 115, 3827-3834 (2010).

2 Klein, T. E., Altman, R. B., Eriksson, N., Gage, B. F., Kimmel, S. E., Lee, M. T. et al.; on behalf of the IWPC. Estimation of warfarin dose using clinical and pharmacogenetic data. N. Engl. J. Med. 360, 753-764 (2009).

3 Sagreiya, H., Berube, C., Wen, A., Ramakrishnan, R., Mir, A., Hamilton, A. et al. Extending and evaluating a warfarin dosing algorithm that includes CYP4F2 and pooled rare variants of CYP2C9. Pharmacogenet. Genomics 20, 407-413 (2010).

4 Perini, J. A., Struchiner, C. J., Silva-Assunção, E., Santana, I. S. C., Rangel, F., Ojopi, E. B. et al. Pharmacogenetics of warfarin: development of a dosing algorithm for Brazilian patients. Clin. Pharmacol. Ther. 84, 722-728 (2008).

5 Suarez-Kurtz, G Population diversity and the performance of warfarin dosing algorithms. Br. J. Clin. Pharmacol. 72, 451-453 (2011).

6 Ansell, J., Hirsh, J., Hylek, E., Jacobson, A., Crowther, M. \& Palareti, G. Pharmacology and management of the vitamin K Antagonists: American College of Chest Physicians evidence-based clinical practice guidelines (8th Edition). Chest 133, 160S-198S (2008).

7 Geisen, C., Watzka, M., Sittinger, K., Steffens, M., Daugela, L., Seifried, E. et al. VKORC1 haplotypes and their impact on the inter-individual and inter-ethnical variability of oral anticoagulation. Thromb. Haemost. 94, 773-779 (2005).

8 Spreafico, M., Lodigiani, C., Leeuwen, Y., Pizzotti, D., Rota, L. L., Rosendaal, F. R. et al. Effects of CYP2C9 and VKORC1 on INR variations and does requirements during initial phase of anticoagulation therapy. Pharmacogenomics 9, 1237-1250 (2008).

9 Cavallari, L. H., Langaee, T. Y., Momary, K. M., Shapiro, N. L., Nutescu, E. A., Coty, W. A. et al. Genetic and clinical predictors of warfarin dose requirements in African Americans. Clin. Pharmacol. Therapeut. 87, 459-464 (2010).

10 Gage, B. F., Eby, C., Johnson, J. A., Deych, E., Rieder, M. J., Ridker, P. M. et al. Use of Pharmacogenetic and clinical factors to predict the therapeutic dose of warfarin. Clin. Pharmacol. Therapeut. 84, 326-331 (2008).

11 Perini, J. A., Struchiner, C. J., Silva-Assuncao, E., Santana, I. S. C., Rangel, F., Ojopi, E. B. et al. Pharmacogenetics of warfaarin: development of a dosing algorithm for Brazilian patients. Clin. Pharmacol. Therapeut 84, 722-728 (2008).

12 Zhu, Y., Shennan, M., Reynolds, K. K., Johnson, N. A., Herrnberger, M. R., Valdes, Jr R. et al. Estimation of warfarin maintainance dose based on VKORC1 $(-1639 \mathrm{G}>\mathrm{A})$ and CYP2C9 genotypes. Clin. Chem. 53, 1199-1205 (2007).

13 Wadelius, M., Chen, L. Y., Lindh, J. D., Eriksson, N., Ghori, M. J., Bumpstead, S. et al. The largest prospective warfarin-treated cohort supports genetic forcasting. Blood 113, 784-792 (2009).

14 Rieder, M., Reiner, A., Gage, B., Nickerson, D., Eby, C., McLeod, H. L. et al. Effect of VKORC1 haplotypes on transcriptional regulation and warfarin dose. N. Engl. J. Med. 352, 2285-2293 (2005).

15 Limdi, N. A., Arnett, D. K., Goldstein, J. A., Beasley, T. M., McGwin, G., Adler, B. K. et al. Influence of CYP2C9 and VKORC1 on warfarin dose, anticoagulation attainment and maintenance among European-Americans and African-Americans. Pharmacogenomics 9, 511-526 (2008)

16 Schelleman, H., Chen, J., Chen, Z., Christie, J., Newcomb, C. W. \& Brensinger, C. M. Dosing algorithms to predict warfarin maintainance dose in Caucasians and African Americans. Clin. Pharmacol. Theraput. 84, 332-339 (2008).

17 Lee, S. C., Ng, S. S., Oldenburg, J., Chong, P. Y., Rost, S., Guo, J. Y. et al. Interethnic variability of warfarin maintenance requirement is explained by VKORC1 genotype in Asian population. Clin. Pharmacol. Theraput. 79, 197-205 (2006).

18 Perini, J. A., Struchiner, C. J., Silva-Assuncao, E. \& Suarez-Kurtz, G. Impact of CYP4F2 rs 108622 on the stable warfarin dose in an admixed patient cohort. Clin. Pharmacol. Theraput. 87, 417-420 (2010).

19 McDonald, M. G., Reider, M. J., Nakano, M., Hsia, C. K. \& Rettie, A. E. CYP4F2 is a vitamin $\mathrm{K} 1$ oxidase: An explanation for altered warfarin dose in carrier of the V433M variant. Mol. Phamarcol. 75, 1337-1346 (2009).

20 Caulifield, T., Fullerton, S. M., Ali-Khan, S. E., Arbour, L., Buchard, E. G., Cooper, R. S. et al. Race and ancestry in biomedical research: exploring the challenges. Genome Med. 1, 8 (2009).

21 Pathare, A. V., Zadjali, S. A., Misquith, R., Alkindi, S. S., Panjwani, V., Lapoumeroulie, C. et al. Warfarin pharmacogenetics: polymorphisms of the CYP2C9, CYP4F2, and VKORC1 loci in a genetically admixed Omani population. Hum. Biol. 84, 67-77 (2012).

22 Voora, D., Koboldt, D. C., King, C. R., Lenzini, P. A., Eby, C. S. et al. A polymorphism in the VKORC1 regulator calumenin predicts higher warfarin dose requirements in African Americans. Clin. Pharmacol. Theraput 87, 445-451 (2010).

23 Takeuchi, F., McGinnis, R., Bourgeois, S., Barnes, C., Eriksson, N., Soranzo, N. et al. A genome-wide association study confirms VKORC1, CYP2C9, and CYP4F2 as principal genetic determinants of warfarin dose. PLoS Genet. 5, 1-9 (2009).

24 Cooper, G. M., Johnson, J. A., Langaee, T. Y., Feng, H., Stanaway, I. B., Schwarz, U. I. et al. A genome-wide scan for common genetic variants with a large influence on warfarin maintenance dose. Blood 112, 1022-1027 (2008). 